\title{
Mapping trends in woody cover throughout Namibian savanna with MODIS seasonal phenological metrics and field inventory data
}

Vladimir R. Wingate ${ }^{1}$, Nikolaus J. Kuhn ${ }^{1}$, Stuart R. Phinn ${ }^{2}$, Cornelis van der Waal ${ }^{3}$

${ }^{1}$ Physical Geography and Environmental Change, University of Basel, Basel, Switzerland

$5 \quad{ }^{2}$ Remote Sensing Research Centre, School of Earth and Environmental Sciences, The University of Queensland, St Lucia, Australia

${ }^{3}$ Agri-Ecological Services, PO Box 28, Omaruru, Namibia

10 Correspondence to: Vladimir R. Wingate(Vladimir.wingate@unibas.ch)

\section{Supplementary material}
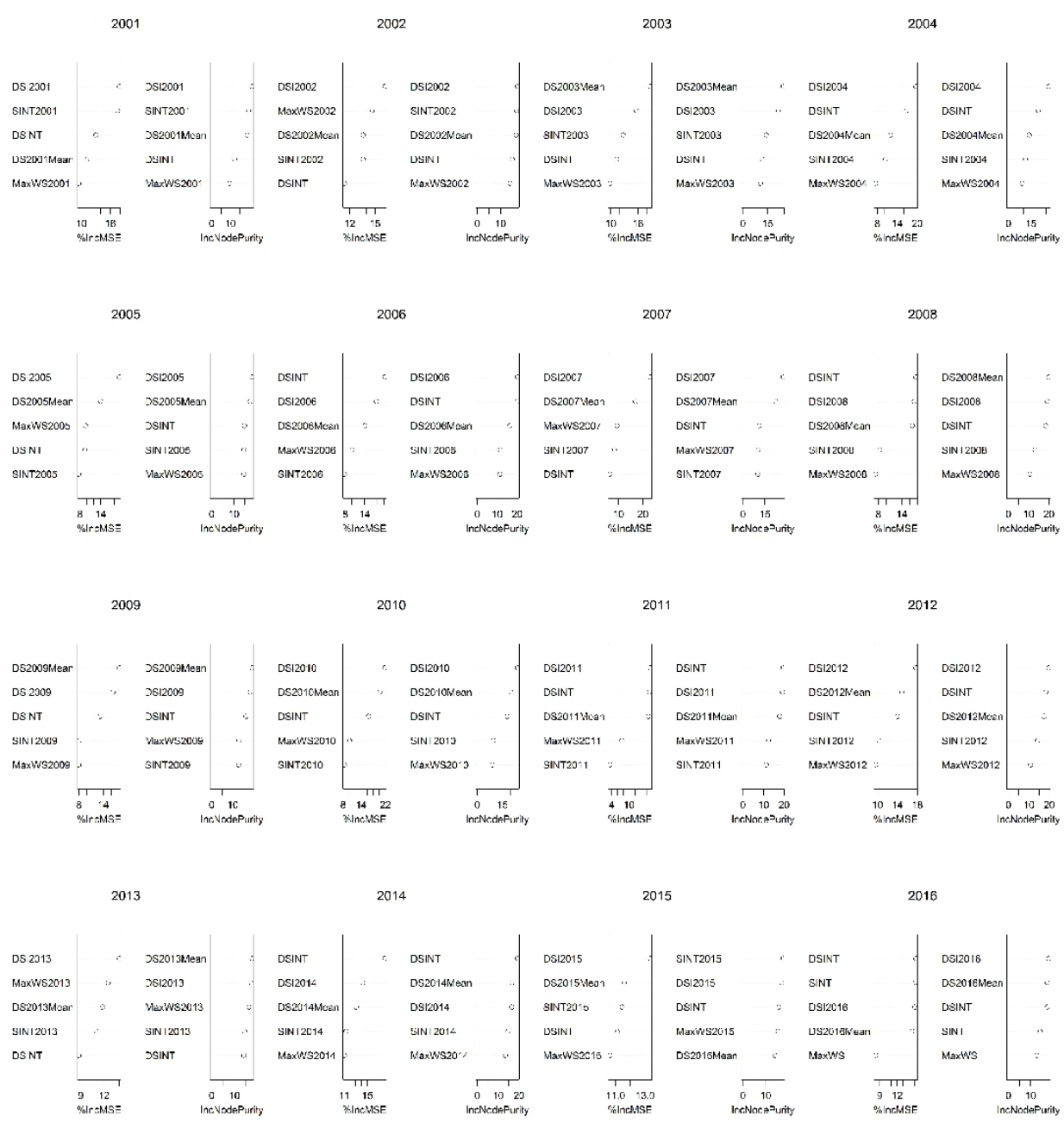
Figure S1. Predictor layer importance is measured using the Mean Standard Error (MSE) as each variable is randomly permuted, and the increase in node purity from each of the splits in the random forest based for each particular variable, as computed by the gini metric.
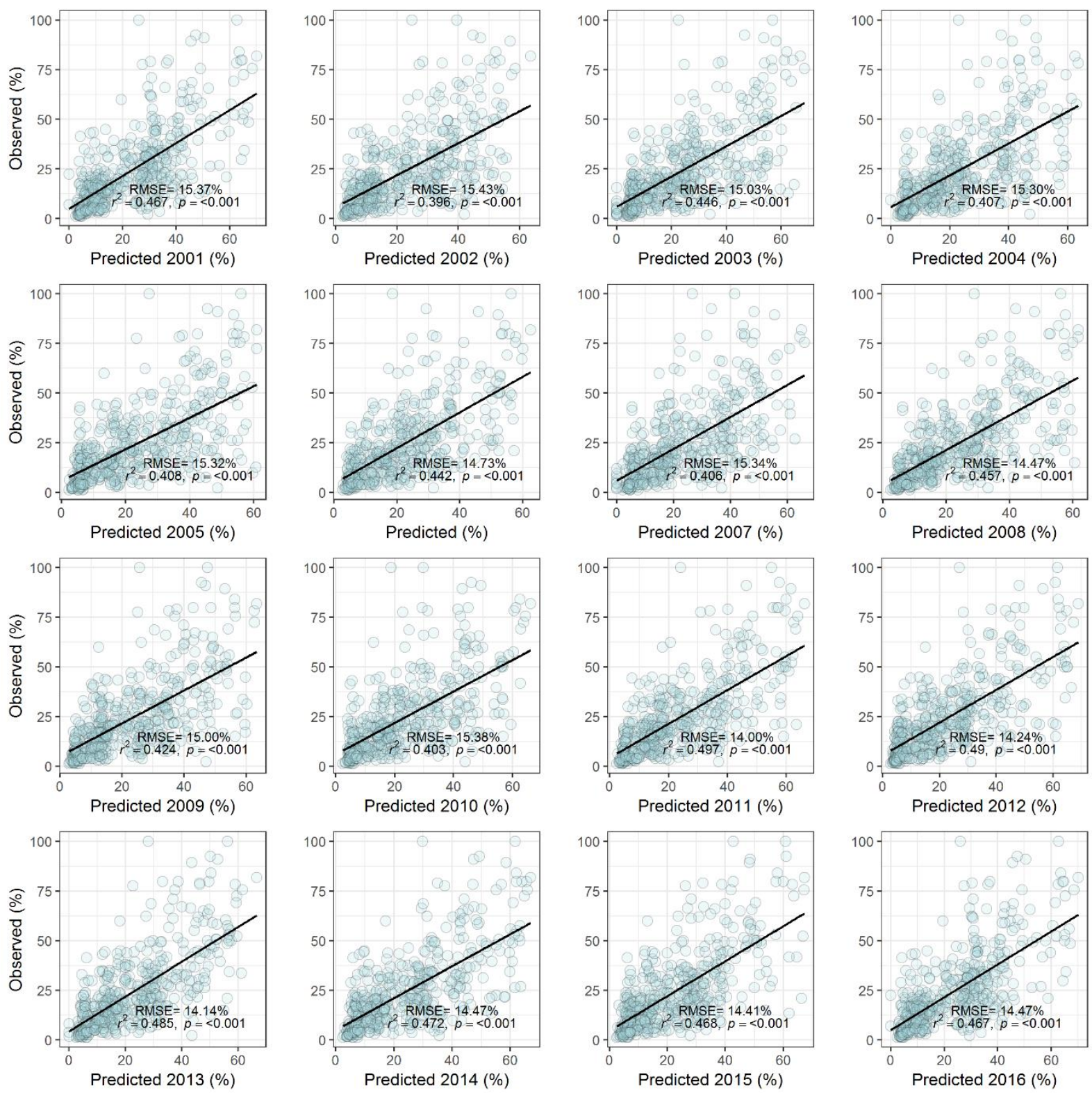

Figure S2. Linear regression of observed and predicted values; between the 2001 and 2016 models, the $R^{2}$ values ranged from 0.4 to 0.5 , and the RMSE ranged from $14.14 \%$ to $15.43 \%$. 

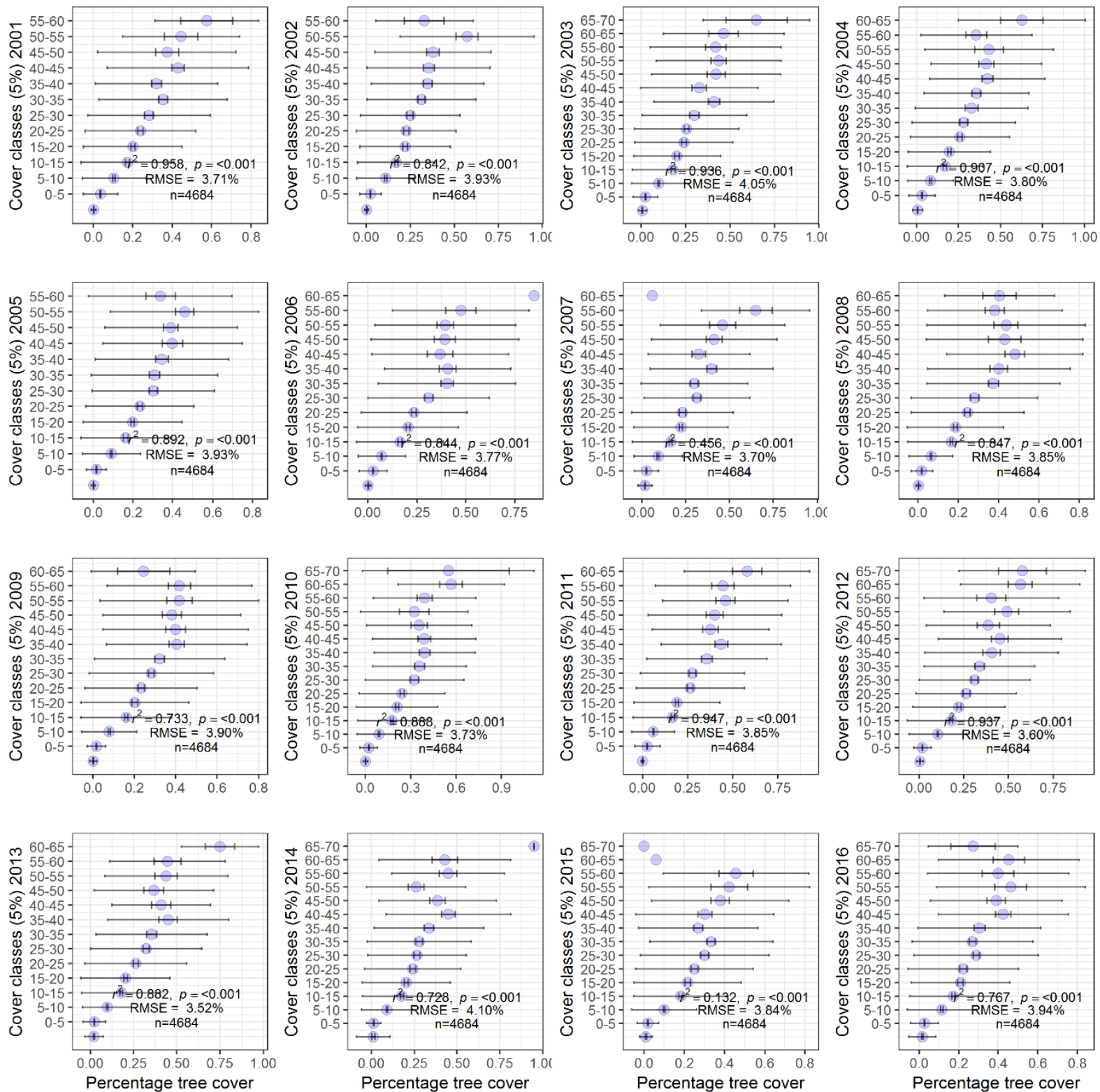

Figure S3. Between the 2001 and 2016 models, the $R^{2}$ values ranged from 0.13 to 0.96 , and the RMSE ranged from 3.52\% to $4.10 \%$. Low $R^{2}$ values are the results of single outlier percentage tree cover $(\%)$ observation within cover classes. For example, for the 2006 model, only a single observed percentage woody cover $(\%)$ sample was identified for the $60-65 \%$ cover class. 

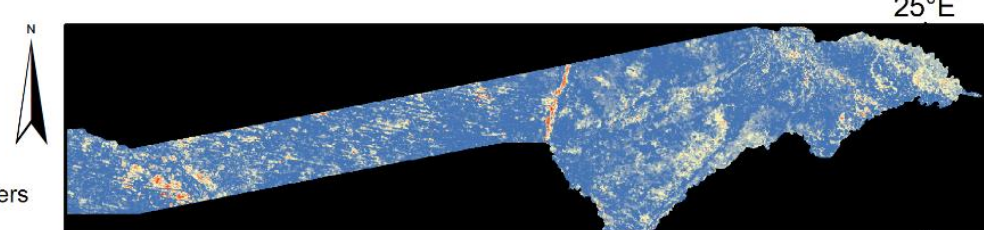

$15^{\circ} \mathrm{E}$

$20^{\circ} \mathrm{E}$

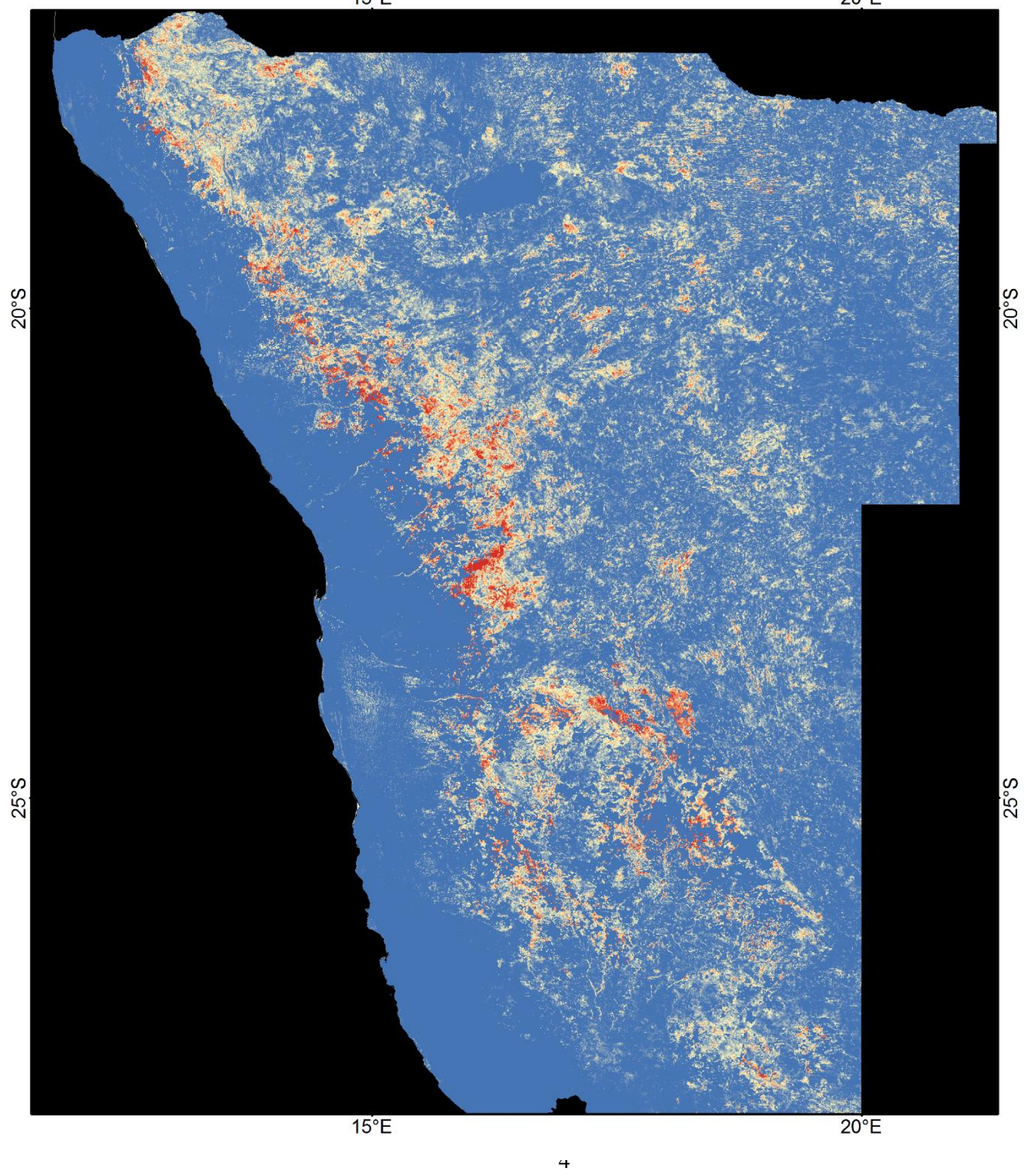


Figure S4. $\mathbf{R}^{2}$ values resulting from a linear regression between mean annual precipitation anomalies (independent) and annual percentage woody cover anomalies (dependent). 